\title{
Traduire
}

Revue française de la traduction

$215 \mid 2007$

La qualité en perspective

\section{Le management de la qualité, facteur et vecteur de confiance}

\section{Elena Pelliser}

\section{(C) OpenEdition}

Journals

\section{Édition électronique}

URL : http://journals.openedition.org/traduire/1254

DOI : 10.4000/traduire. 1254

ISSN : 2272-9992

\section{Éditeur}

Société française des traducteurs

\section{Édition imprimée}

Date de publication : 1 décembre 2007

Pagination : 5-13

ISSN : 0395-773X

\section{Référence électronique}

Elena Pelliser, "Le management de la qualité, facteur et vecteur de confiance », Traduire [En ligne], 215 | 2007, mis en ligne le 01 décembre 2007, consulté le 02 mai 2019. URL : http:// journals.openedition.org/traduire/1254; DOI : 10.4000/traduire.1254 


\title{
Le management de la qualité, facteur et vecteur de confiance
}

\author{
Elena Pelliser
}

Rien ne génère davantage la polémique dans la sphère des traducteurs que la notion de qualité : nous prônons et recherchons tous la qualité maximale dans notre travail, mais il arrive que, malgré tout le soin apporté à une traduction, le client ne soit pas entièrement satisfait du produit final, car son impression de ce que devrait être la qualité de la traduction remise n'est pas forcément celle des professionnels que nous sommes. Le traducteur avance des arguments de technicien (difficulté du sujet, mauvaise qualité de l'original, problèmes d'informatique...), le client quant à lui formule des reproches de consommateur (délai non tenu, vocabulaire maison non utilisé, présentation non-conforme...) ; le dialogue devient alors difficile, précisément parce que la traduction n'est pas une science exacte, qu'elle relève davantage des sciences humaines. Or, le fait que la qualité perçue par le client soit basée sur une impression et non sur des connaissances professionnelles ne veut pas dire pour autant que cette impression n'est pas légitime. C'est cette difficulté de compréhension que le " management de la qualité » (1), en tant que cadre de comportements et de compétences qui permet de bâtir la confiance (facteur) et de la véhiculer (vecteur), peut contribuer à pallier ou résoudre, en donnant aux traducteurs et à leurs clients un langage commun, et en précisant ce que sont les attentes et les possibilités de chacun.

(1) Cette expression émane de l'AFNOR elle-même (voir en particulier la notice explicative in Qualité et systèmes de management ISO 9000). Elle entend prendre en compte le fait que nous sommes passés de la gestion (chiffres et faits) au management (des hommes, des comportements et des ressentis). 


\section{La Qualité à travers les siècles}

Parce que la qualité (gage de confiance et de respect) accompagne en filigrane tout acte impliquant une transaction, elle est attestée depuis des siècles et a évolué parallèlement aux us et coutumes commerciaux.

La première trace remonte au code d'Hammourabi (roi de Babylone au XVIII siècle av. J.-C.) qui, en 282 arrêts, pose les droits et devoirs des diverses catégories composant son peuple. L'arrêt 233 concerne l'obligation du maçon de refaire un mur mal bâti. Les Phéniciens sont ensuite les premiers connus pour avoir mis en place une "action corrective " brutale basée sur un "contrôle statistique " à vue, en coupant la main de qui réalisait à plusieurs reprises des produits d'une qualité insuffisante. Le serment d'Hippocrate s'apparente tout à fait à une Charte qualité engageant le médecin à tout faire pour soigner son patient et, en corollaire, à entretenir et parfaire ses connaissances en permanence. L'époque médiévale pousse très loin le concept de label et d'étalons de mesure, et les premières méthodes d'échantillonnage destinées à contrôler le titre et le poids des monnaies frappées pour le compte du roi d'Angleterre sont appliquées dès le XIIe siècle. Les productions artistiques aussi répondent à un véritable cahier des charges : le contrat établi en 1516 entre les Moniales de Bruges et Raphaël pour l'exécution d'un Couronnement de la Vierge comporte 11 clauses relatives aux dimensions, à la qualité des couleurs et aux délais de livraison (la clause 7 prévoyant que Raphaël serait tenu de réparer à ses frais tout dommage intervenu durant le transport de l'œuvre de Rome à Bruges).

Nous sommes face à une conception très " qualitative " de la qualité, marquée par le lien direct et en face-à-face entre le producteur et le client, fondée sur l'engagement de la responsabilité personnelle et sur un transfert des connaissances qui s'accompagne d'un cheminement spirituel. Cette approche va perdre peu à peu de l'importance à partir du XVIIe siècle et des débuts de l'industrie, même s'il reste encore des " ouvriers" (qui œuvrent) gardiens de traditions séculaires, et passer dans l'ombre pendant quatre siècles au profit d'une qualité de plus en 
plus « quantitative ", pour mieux revenir sur le devant de scène depuis une dizaine d'années. Un tableau synoptique permettra de mieux comprendre comment, de cette approche qualitative qui correspond tout à fait à l'esprit de la traduction, on a pu en arriver à une approche quantitative (mesures, chiffres, ...) qui, pour nous, s'apparente parfois à la simple lettre de la qualité.

Du code d'Hammourabi au XVIIe siècle : artisanat et contrôle

- Formalisation de la notion de qualité.

- Passage de la "qualité = beauté " à la "qualité = utilité ".

- Mise en place d'un système structuré de responsabilité du producteur visà-vis du client et de la corporation de rattachement $=>$ responsabilité collective et autodiscipline de marché.

- Transfert de connaissances accompagné d'une démarche spirituelle.

De 1800 à 1940 : maîtrise de la qualité des productions industrielles Deux objectifs : compétitivité, rentabilité. Trois conséquences : taylorisme, contrôle et inspection, avec pour corollaire la désaffection pour le travail de plus en plus déshumanisé et la prise en charge du contrôle qualité par les pouvoirs publics (notamment en métrologie et normalisation).

De 1940 à 1960 : Deuxième Guerre mondiale, nouvelles technologies Dans l'armement : besoin d'efficacité à grande échelle pour alimenter en armes les soldats au front.

Dans le secteur civil après guerre : automobile, nucléaire et avionneurs civils - pour sécuriser leurs produits et fiabiliser les processus de production. Développement de l'autocontrôle et de la fonction qualité en entreprise.

De 1960 à 1990 : la qualité comme arme économique Le Japon s'appuie sur les méthodes de qualité pour conquérir des marchés. Développement des preuves contractuelles de qualité sous la pression des consommateurs - certificats, labels, étiquetage, procès-verbaux d'essais etc. - et harmonisation communautaire par des Directives.

De 1990 à aujourd'hui : reconnaissance accrue de la qualité dans les services. La qualité est la clé de voûte car elle touche toute la chaîne : marketing, $\mathrm{RH}$, finances, contrôle de gestion, ventes, relations clients/fournisseurs, stratégie, leadership ... 
La définition de la qualité qui permet de relier les exigences de traducteurs artisans d'art face à leur métier et les attentes de clients consuméristes face à une prestation est donnée par l'ISO 8402 (version 1994) :

Ensemble des propriétés et caractéristiques d'un produit ou service qui lui conferent l'aptitude à satisfaire les besoins exprimés ou implicites [du client].

En d'autres termes, il s'agit de passer intellectuellement de la notion simple de traduction type " texte remis sur papier ou sur support électronique " à la notion complexe de prestation de services de traduction, où l'acte de traduction n'est qu'un des éléments de la prestation, ce qui entraîne diverses conséquences et notamment de devoir identifier précisément quelle est la qualité attendue par le client (et, à la livraison, la qualité perçue par celui-ci) et quelles seront les " parties prenantes " de la traduction et leurs besoins explicites et implicites afin de pouvoir y répondre au mieux.

\section{La Qualité, facteur de confiance}

Comme pour toute activité exercée à titre permanent, il est intéressant de fiabiliser la production au maximum en sécurisant les procédés et les processus ; il s'agit pour cela de :

- Faire correctement le travail : maîtrise des connaissances et professionnalisme dans les comportements, y compris pour la tenue des livres comptables et le suivi des clients.

- Vérifier qu'on l'a fait correctement : contrôle et révision(s) - il arrive encore trop souvent que les traducteurs ne trouvent pas le temps de faire la dernière révision qui aurait permis de rendre un texte impeccable, ce qui est dommage pour leur image de marque.

- Dire ce qui est proposé : par exemple, si vous avez un site personnel, un calendrier en ligne des créneaux libres facilitera les 
démarches et vous évitera de vous laisser convaincre d'accepter par faiblesse le texte de trop que vous ne pourrez assumer ensuite.

- Dire ce qui est possible : accepter un texte dépassant vos connaissances ou qui se révèle impossible à faire dans les délais contractuels acceptés peut coûter très cher en réputation et en responsabilité civile.

- Améliorer en permanence ce qui est possible : tirer - lucidement les leçons de ses déboires, ou de ses grosses frayeurs !

- Connaître ses clients et/ou ses fournisseurs : pour mieux comprendre les demandes implicites, proposer des services annexes ou anticiper leurs besoins etc.

- CAPITALISER SUR SON EXPÉRIENCE INTELLECTUELLE : parce que le meilleur ami d'un traducteur, c'est son cerveau, il faut enrichir ses connaissances, se former, se semi-spécialiser le cas échéant, échanger avec des collègues traducteurs ou d'autres professionnels, sortir de son bureau, construire ses glossaires de manière ordonnée, etc.

Cette démarche vous permet de créer et de conforter votre propre capital confiance de professionnel, puisque 1) vous savez ce que vous proposez comme services et pouvez donc vous montrer à juste titre sélectif sur les contrats que vous acceptez, 2) vous pouvez fidéliser un " fonds de clientèle et/ou de fournisseurs " régulier avec qui vous allez progressivement bâtir non plus un simple réseau, mais bel et bien un "partenariat de fait".

\section{La Qualité, vecteur de confiance}

En affaires, on a rarement une deuxième chance de faire une première bonne impression. La Qualité, parce qu'elle induit et bâtit votre confiance en vous, vous aide à transformer plus facilement un contact en une relation de travail. Par ailleurs, savoir ce que l'on propose comme services (spécialité et/ou langues rares) et les créneaux sur lesquels on est le plus à l'aise constitue un énorme avantage marketing, 
car cela permet de fidéliser les clients autour de ce pôle de compétences. En outre, parler le même langage est un atout de taille, car la plupart des entreprises sont passées à un management participatif reposant sur les principes de gestion de la qualité et vous ferez d'autant mieux entendre votre argumentaire que vous vous réfererez à des concepts communs. Or, plus vos clients et fournisseurs sont fidélisés, plus vous aurez pérennisé votre propre activité.

On voit de plus en plus fréquemment des agences qui sont certifiées ISO ou proposent une Charte qualité, une assurance qualité... Lorsqu'on travaille en indépendant, il est plus difficile de faire cette démarche, mais il faut savoir qu'elle n'est pas impossible, même si cela suppose une forte charge administrative et logistique en amont avant de pouvoir être certifié (manuel qualité, obligations documentaires et comptables, audits réguliers de qualité et de maintien de la certification...).

Sans en arriver à des solutions lourdes de ce type, il est néanmoins possible d'adopter quelques sains principes de management dans une optique de qualité : la bonne tenue des comptes n'est pas une activité accessoire à repousser à plus tard ; informez-vous sur vos principaux clients, leurs activités, leurs contraintes (comptables, techniques, réglementaires ou autres); si vous avez des "fournisseurs", autrement dit si vous déléguez des textes à d'autres collègues, supervisez leur travail, testez les nouveaux entrants qui vous contactent, harmonisez au maximum le couple traduction/traducteur ; intéressez vos «fournisseurs " à votre activité, intégrez-les, mettez en commun des informations, glossaires, faites en sorte qu'ils se sentent partie prenante d'une bonne prestation.

\section{La Qualité, comment faire ?}

À cela, la plus commune des réponses : avancer un peu à la fois, et, pour les novices qui souhaitent s'informer d'abord, il est vivement conseillé de commencer par consulter le fascicule de documentation 
FD-X 50-179 (in Qualité et systèmes de management ISO 9000 $\left.A F N O R^{(2)}\right)$ qui donne une structure et une démarche pratique en même temps que les fondamentaux de la qualité. Pour les approches plus conceptuelles et "philosophiques", il conviendra de se familiariser avec des auteurs tels que Deming, Crosby et Juran.

Vous avez sans doute entendu parler d'un certain nombre d'outils sans savoir que ceux-ci sont liés à la qualité : knowledge management et capital intellectuel, orientation client, diversité et management interculturel, compétence collective, gestion des compétences, création de valeur, cercles de réflexion, sessions de remue-méninges, cercles de qualité, qualité dans les services (Servuction). Si vous souhaitez passer à une démarche de qualité, il serait bon de vous familiariser avec ces outils pour mieux comprendre comment les mettre à profit dans votre activité à court, moyen et long terme. Enfin, parce que le management de la qualité intègre les principaux courants et idées phares d'un demisiècle de science de gestion et qu'il est utile de s'y référer pour comprendre en quoi la Qualité est une solution intéressante, une liste d'ouvrages de référence est indiquée en annexe.

\section{Conclusions}

La Qualité est une démarche :

- Qui repose sur la compétence et son développement, au niveau individuel et collectif.

- Qui postule l'amélioration permanente.

- Qui prône les solutions gagnant-gagnant.

- Qui fait une large place aux transferts et à l'enrichissement des connaissances.

- Qui inscrit l'individu, la prestation, l'activité dans la confiance, seul gage d'une relation à long terme.

(2) ISBN 2-12-215071-8, AFNOR 2001, pages 253 et suivantes, mais tout l'ouvrage est une mine d'informations. 
À ce titre, parce que la qualité associe les atouts d'une approche humaniste du cœur de métier à des outils de gestion qui ont fait leurs preuves, le management de la qualité est un cadre de réflexion et d'opération intéressant dans lequel inscrire des activités de traduction, qu'elles soient menées en indépendant, en salarié ou en société.

Elena Pelliser est réviseur au sein du Service de la traduction française générale au Conseil de l'Europe, où elle s'intéresse plus particulièrement aux questions de stratégie et de management de la qualité. Doublement diplômée de l'ESIT (1988 et 1989), puis de HEC en 1994, elle est également titulaire d'un Mastère Gestion de la Qualité de l'Université de Strasbourg (2005). Elle exerce en tant que traductrice depuis 19 ans.

elena.pellliser@coe.int

\section{Annexe : bibliographie raisonnée}

Cette très courte bibliographie regroupe cinq ouvrages de base pour démarrer :

AFNOR, Qualité et systèmes de management ISO 9000, Paris, AFNOR, 2001.

L'ensemble du recueil, de lecture accessible même pour le novice, expose de manière progressive et cohérente l'environnement et les textes normatifs, puis les notices explicatives. Les notices FD-X-50179, 172 et suivantes déroulent en détail la démarche pour identifier les besoins des clients et mesurer la satisfaction du client. Le monde de l'entreprise fonctionne de plus en plus (notamment pour les grands groupes internationaux) selon cette logique.

Jean Brilman, Les meilleures pratiques de management, Paris, Éditions d'Organisation, 2003.

Les chapitres 4, 8, 13, 14 et 15 en particulier donnent une vision synthétique et complémentaire des enjeux de la relation client, de la gestion de valeur, de la TQM et des réseaux. Excellente bibliographie en complément de lecture. 
Daniel Gouadec, Profession : traducteur, Paris, La Maison du Dictionnaire, 2002.

Cet ouvrage qui n'est plus à présenter pose les problématiques de fond de la profession et des métiers de traducteur. À lire, ou à relire, dans une démarche préliminaire à toute mise en place d'un système d'assurance qualité.

J.-P. Hubérac, Guide des méthodes de la qualité, 2e édition, Paris, Laurent du Mesnil Éditeur, Maxima, 2001.

On trouvera dans cet ouvrage incontournable une gamme d'outils qui ont fait leurs preuves, pour choisir en connaissance de cause ceux qui conviennent le mieux à une démarche qualité personnalisée. Chaque outil présenté est assorti d'une bibliographie succincte pour explorer le sujet plus avant.

Pierre Vandeville et Christine Gambier, Conduire un audit de qualité, Paris, AFNOR, 1995.

Méthodologie et techniques de conduite d'un audit de la qualité ; très structuré, mais assez austère, il permet toutefois de mieux percevoir par l'exemple la philosophie d'une démarche de qualité réussie. 